\title{
Aplicaciones clínicas del ayuno intermitente
}

\author{
Clinical applications of intermittent fasting \\ Aplicações clínicas do jejum intermitente
}

\author{
Marisa Canicoba ${ }^{1 *}$ \\ Recibido: 9 de julio de 2020. Aceptado para publicación: 20 de julio de 2020 \\ Publicado en línea: 31 de julio de 2020 \\ https://doi.org/10.35454/rncm.v3n2.174
}

\section{Resumen}

El objetivo de esta revisión es proporcionar una visión general del ayuno intermitente, resumir la evidencia y discutir los mecanismos fisiológicos por los cuales el ayuno intermitente podría conducir a un beneficio para la salud.

En las últimas décadas, varias líneas de evidencia científica respaldan la implementación de este tipo de ayuno a través de los beneficios sobre la regulación metabólica de los efectos sobre el ritmo circadiano, el microbioma intestinal, pérdida de peso, diabetes mellitus, esteatosis hepáticas, enfermedad cardiovascular, entre otras patologías. Sin embargo, se requieren estudios aleatorizados para determinar si el ayuno intermitente, como un estilo de vida, es beneficioso para la salud.

Palabras clave: ayuno intermitente, ritmo circadiano, microbiota intestinal.

\section{Abstract}

The objective of this review is to provide an overview of intermittent fasting, summarize the evidence, and discuss the physiological mechanisms by which intermittent fasting could lead to health benefits.

In recent decades, several scientific publications have supported the implementation of this type of fasting as a result of its beneficial effects on circadian rhythm and metabolism, intestinal microbiome, weight loss, diabetes mellitus, liver steatosis, and cardiovascular disease. Yet, randomized controlled studies are required to determine the health benefits of fasting as a part of lifestyle.

Keywords: Intermittent fasting; Circadian rhythm; Gut microbiota.

\section{Resumo}

O objetivo desta revisão é fornecer uma visão geral do jejum intermitente, resumir as evidências e discutir os mecanismos fisiológicos pelos quais o jejum intermitente pode conduzir a um benefício para a saúde.

Nas últimas décadas, várias linhas de evidências científicas apoiam a implementação desse tipo de jejum através dos benefícios na regulação metabólica dos efeitos no ritmo circadiano, no microbioma intestinal, na perda de peso, na diabetes mellitus, na esteatose hepática, na doença cardiovascular, entre outras patologias. No entanto, são necessários estudos randomizados para determinar se o jejum intermitente, como estilo de vida, é benéfico para a saúde.

Palavras-chave: jejum intermitente, ritmo circadiano, microbiota intestinal.
Hospital Nacional A. Posadas, Universidad de Buenos Aires, Buenos Aires, Argentina.
*Correspondencia: Marisa Canicoba marisa.canicoba@gmail.com

\section{INTRODUCCIÓN}

La restricción de energía junto con una intervención integral sobre el estilo de vida se considera la piedra angular del tratamiento de la obesidad; sin embargo, este enfoque produce una pérdida de peso moderada ${ }^{(1)}$.
Nuestros ancestros humanos no consumían alimentos regularmente, ni tampoco tenían una vida sedentaria, en cambio, estaban ocupados en la adquisición de alimentos. Es probable que Hipócrates sea uno de los primeros defensores del ayuno con fines médicos, ya que recomendaba ayunar durante la enfermedad. 
En la actualidad, las recomendaciones para disminuir de peso incluyen consejos para comer regularmente incorporando seis comidas diarias. Aunque recientemente ha aumentado el interés en identificar estrategias alternativas de pérdida de peso que impliquen restringir la ingesta de energía durante ciertos períodos del día o prolongar el intervalo de ayuno entre comidas. Por esto, en las últimas décadas, el ayuno ha aumentado su popularidad como método para la prevención y promoción de la salud.

Cada día surgen más estudios sobre su eficacia en la prevención y el tratamiento en enfermedades crónicas como: obesidad, diabetes, cardiovasculares, inflamatorias y degenerativas, durante la quimioterapia del cáncer, problemas asociados al envejecimiento. Un artículo de revisión publicado en diciembre de $2019^{(2)}$ en la revista New England Journal of Medicine, realizado por el neurocientífico de la Universidad de Johns Hopkins, Mark Mattson, concluye que el ayuno intermitente (AI) cuenta con evidencia científica y describe que esta medida podría ser parte de un estilo de vida saludable. Por lo tanto, el objetivo de esta revisión es proporcionar una visión general del ayuno intermitente, resumir la evidencia sobre sus aplicaciones clínicas, y discutir los mecanismos fisiológicos por los cuales el ayuno intermitente podría conducir a un beneficio para la salud.

Los estudios de ayuno intermitente se buscaron a través de MEDLINE (Pubmed) y el Registro Cochrane Central de ensayos controlados. Se utilizaron los siguientes términos de búsqueda en todas las combinaciones posibles: "ayuno intermitente", "aplicaciones clínicas", "efectos metabólicos", "beneficios para la salud". Se incluyeron artículos que cumplían con los siguientes criterios de inclusión para estudios en humanos: 1 . revistas en inglés, 2 . estudios de diseño aleatorizados, revisiones sistemáticas, estudios randomizados controlados, en adultos (edad $\geq 18$ años), 3. informe clínico con resultados de interés que evaluaron los efectos del ayuno en los resultados de eventos clínicos; y 4. se examinaron en forma independiente todos los estudios identificados y se realizó la inclusión de aquellos que se consideraron de interés.

\section{FISIOLOGÍA DEL AYUNO INTERMITENTE}

El ayuno intermitente (AI) puede definirse como una abstinencia voluntaria de alimentos y bebidas por períodos específicos y recurrentes. Es importante determinar a qué tipo de ayuno nos referimos. En el ayuno intermitente, la participación del paciente es voluntaria. Es decir, el AI, no debe confundirse con inanición, un estado de deficiencia nutricional crónica que no es voluntaria ni controlada, y que puede culminar en insuficiencia orgánica y muerte ${ }^{(1)}$.

El ayuno induce la alteración coordinada de mecanismos metabólicos y transcripcionales (Figura 1). Luego de 12 a 36 horas de ayuno, el cuerpo humano entra en un estado fisiológico de cetosis caracterizado por niveles bajos de glucosa en sangre, disminución del depósito de glucógeno en hígado, yla producción hepática de cuerpos cetónicos (CC) derivados de la grasa, o cetonas, que sirven como fuente importante de energía para el cerebro ${ }^{(2)}$. El hígado es el sitio primario de la cetogénesis, pero los astrocitos cerebrales también generan cetonas. Luego de varios días de iniciar el ayuno, las cetonas se convierten en la fuente de combustible preferida del cerebro (beta-

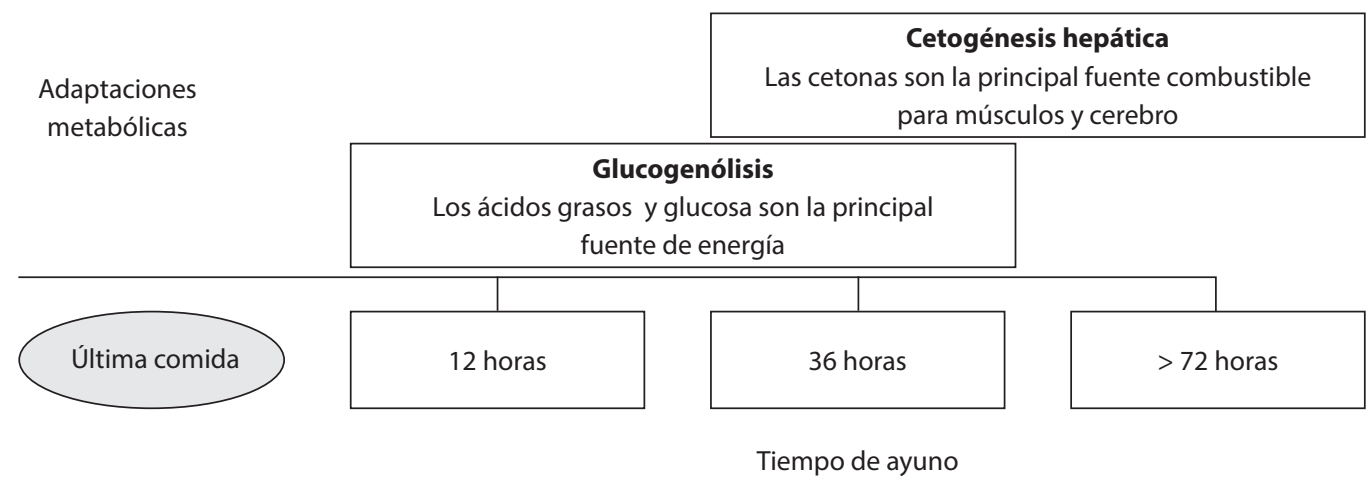

Figura 1. Adaptaciones metabólicas según la fase de ayuno. 
hidroxibutirato y acetoacetato), proporcionando hasta $70 \%$ de sus necesidades energéticas ${ }^{(3)}$. Las cetonas constituyen una fuente de energía más eficiente en los músculos y posiblemente en el cerebro, mejorando la bioenergética y la actividad conectiva de las neuronas.

Sin embargo, las cetonas son más que una fuente de energía para las neuronas: la cetona beta-hidroxibutirato (BHB), por ejemplo, cumple importantes funciones de señalización al inducir la transcripción de derivados del factor neurotrófico derivado del cerebro (FNDC). El FNDC es un regulador fundamental de la función neuronal que estimula la biogénesis de la mitocondria, mantiene la estructura sináptica, estimula la producción y supervivencia de nuevas neuronas y aumenta la resistencia de éstas a lesiones y enfermedades ${ }^{(1)}$.

Además de BHB y FNDC, el ayuno induce la expresión de un regulador de mitocondrias, la proteína "Peroxisome proliferator-activated receptor $\gamma$ co-activator $1 \alpha$ " (PPARGC-1-alpha o PGC1 $\alpha$ ) que juega un papel importante en la modulación de genes relacionados con el metabolismo de los carbohidratos y los ácidos grasos entre otras muchas funciones $^{(1)}$ (Figura 2).

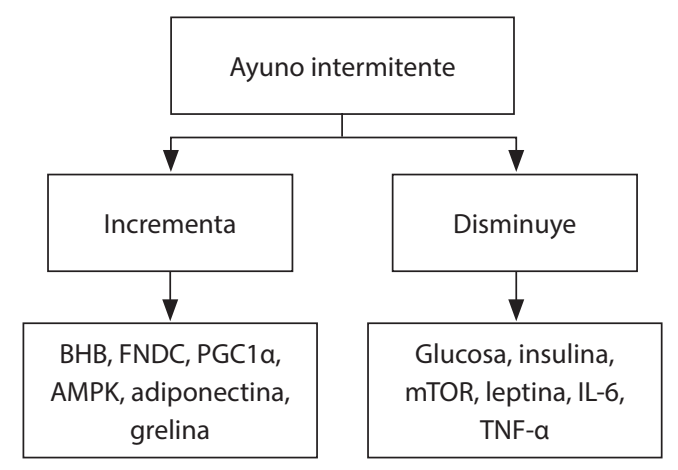

Figura 2. Mecanismos metabólicos y transcripcionales inducidos por el ayuno. Abreviaturas: BHB: cetona betahidroxibutirato, FNDC: factor neurotrófico derivado del cerebro, PGC1a: peroxisoma activado por el proliferador receptor $y$ coactivador 1, AMPK: proteína quinasa activada por AMP, mTOR: complejo sensible a rapamicina, IL-6: Interleuquina 6, TNF- a: Factor de necrosis tumoral a.

El ayuno suprime la inflamación reduciendo la expresión de citocinas proinflamatorias como la Interleuquina 6 (IL-6) y el Factor de Necrosis Tumoral $\alpha(\text { TNF- } \alpha)^{(4,5)}$. El ayuno también:

1. Influye en los procesos de síntesis y degradación celular al impedir la acción del complejo sensible a rapamicina (mTOR, por sus siglas en inglés) que puede causar la destrucción de las células cancerosas ${ }^{(1,3)}$.
2. Eleva la proteína quinasa activada por AMP (AMPK), lo que limita el consumo de nutrientes ${ }^{(3)} y$ se promueve la autofagia. Una variedad de estímulos de estrés inducen autofagia, el ayuno y la restricción calórica se consideran una intervención óptima para mejorar la salud y aumentando la longevidad ${ }^{(6)}$.

3. Influye en el metabolismo de los lípidos al alterar las actividades hormonales de la leptina, la adiponectina y grelina. La leptina se asocia a un estado proinflamatorio, mientras que la adiponectina a una mayor sensibilidad a la insulina. La grelina puede estimular la neurogénesis. La leptina disminuye pero la adiponectina y grelina aumentan, alteraciones que probablemente sean beneficiosas para la bioenergética de las neuronas y el mantenimiento de vías neuronales ${ }^{(3)}$.

\section{RITMO CIRCADIANO}

El organismo ha desarrollado un ritmo circadiano endógeno para garantizar que los procesos fisiológicos se cumplan en horarios óptimos ${ }^{(7)}$. Los ritmos circadianos optimizan la fisiología y la salud al coordinar temporalmente la función celular, tisular, y el comportamiento. Los patrones de ayuno de alimentación son una señal externa que influye profundamente en la solidez de los ritmos biológicos diarios.

En los humanos tiene un impacto en el metabolismo, por ejemplo: la sensibilidad a la insulina disminuye a lo largo del día y durante la noche, esto se debe, en parte, al ritmo circadiano de la secreción de insulina y a la acción de la hormona del crecimiento que mejora la insulina, cuyas concentraciones pulsátiles aumentan por la noche ${ }^{(8)}$. Las respuestas posprandiales de insulina y glucosa aumentan a lo largo de la edad y la noche. Por lo tanto, las comidas que se ingieren por la noche se asocian a una mayor exposición posprandial a la glucosa y a la insulina que las comidas con contenido similar que se consumen durante el día, lo que aumenta los niveles de hemoglobina glicosilada y el riesgo de diabetes tipo 2 con el tiempo ${ }^{(9)}$. Por lo tanto, el ritmo circadiano puede ayudar a reducir la carga de las enfermedades crónicas ${ }^{(10)}$.

\section{CARACTERÍSTICAS DE LAS DIETAS DE AYUNO INTERMITENTE}

Tres parámetros caracterizan un régimen de ayuno:

1. La intensidad de la restricción de alimentos y bebidas: es decir cantidad, tipo de alimentos y bebidas 
que pueden permitirse durante los períodos de ayuno. La intensidad de un período de ayuno varía desde la omisión completa de comida y bebida (un ayuno "puro") a una ingesta mínima de comidas específicas destinadas a mantener el estado metabólico.

2. La frecuencia de los períodos de ayuno

3. La duración de los períodos de ayuno.

El régimen de ayuno ideal depende del estilo de vida y la tolerabilidad de cada individuo.

El AI no consiste en dejar de comer, sino hacerlo en intervalos de tiempos específicos. Por ejemplo, establecer intervalos de 12 horas donde se organicen las comidas y 12 horas donde se ayune, aunque algunos estudios proponen un ayuno de 16 horas y la ingesta durante 8 horas restantes.

Las dietas de ayuno más conocidas se dividen en:

- Ayuno intermitente (AI): ayuno de 2 - 3 días por semana o también conocida como dieta 5:2 que implica restricción de energía durante dos días no consecutivos por semana y comer sin restricciones durante los otros 5 días.

- Ayuno alterno (AA): implica días durante los cuales no se consumen calorías y días de alimentación ad libitum.

El AA fue efectivo para la pérdida de peso y salud cardiovascular en adultos con sobrepeso y peso normal. Los indicadores de salud se hacen evidentes dentro de las 2 a 4 semanas luego del inicio del AA, evidenciándose una reducción de las concentraciones de colesterol total y triacilglicerol, disminución de la frecuencia cardíaca, presión arterial y una mejor respuesta cardíaca al infarto de miocardio ${ }^{(11)}$.

\section{BENEFICIOS DEL AYUNO INTERMITENTE}

Una de las razones por las que se adopta este tipo de alimentación es el cambio metabólico que produce sobre:

- Pérdida de peso

- Mejora la regulación de la glucosa y la sensibilidad de insulina, presión arterial, frecuencia cardíaca, eficacia del entrenamiento de resistencia, pérdida de grasa abdominal

- El organismo cambia el uso de glucosa como fuente de combustible por ácidos grasos y cuerpos cetónicos aumentando la resistencia ante el estrés oxidativo y reduciendo los marcadores de inflamación sistémica asociados a la aterosclerosis.

\section{MICROBIOTA GASTROINTESTINAL}

Muchas funciones del tracto gastrointestinal tienen ritmos circadianos de sueño - vigilia, por ejemplo, el vaciado gástrico y el flujo sanguíneo son mayores durante el día que durante la noche y las respuestas metabólicas son mayores a una carga de glucosa más lenta por la tarde que por la mañana. Con base en esto, es plausible que un perfil circadiano alterado crónicamente pueda afectar la función gastrointestinal y perjudicar el metabolismo y la salud ${ }^{(5)}$.

Los cambios cíclicos en el intestino, el microbioma resultante de la alimentación diurna y los ritmos de ayuno contribuyen a la diversidad del intestino, por lo que el AI puede influir directamente en la microbiota intestinal. Un período de ayuno prolongado (reposo intestinal) también podría reducir la permeabilidad intestinal y, mitigar la endotoxemia posprandial y la inflamacion sistémica que se encuentran elevadas en el paciente obeso. ${ }^{(5,12,13)}$.

Nuevas investigaciones deberán evaluar el impacto en la salud de los regímenes de ayuno en la microbiota humana que tienen el potencial de hacer contribuciones importantes a este campo.

\section{APLICACIONES CLÍNICAS DEL AYUNO INTERMITENTE}

\section{Consideraciones y limitaciones}

\section{Diabetes Mellitus}

Dos estudios mostraron que el AI (4:3) ayuno de 24 horas, 3 veces por semana revirtió la resistencia a la insulina en pacientes con prediabetes o diabetes tipo 2 , reducir los niveles de hemoglina glicosilada, el estrés oxidativo y el apetito $^{(14,15)}$.

\section{Sobrepeso y obesidad}

Recientemente se publicaron dos revisiones en pacientes adultos con sobrepeso u obesidad y encontraron que el AI es tan eficaz para perder peso como las dietas estándar ${ }^{(16,17)}$.

Varady et al. ${ }^{(18)}$ observaron que el AA fue efectivo para la pérdida de peso y salud cardiovascular en adultos con sobrepeso y peso normal. Concluyeron que una restricción de calorías diaria versus una restricción intermitente (24 horas ad libitum alternada con 24 horas de restricción de alimentos completa o parcial) son igualmente efectivas para disminuir de peso y la masa grasa. 
En un ensayo aletaorizado realizado por Trepanowski et al. ${ }^{(19)}$ concluyeron que el AA no produjo una adherencia superior, pérdida de peso, mantenimiento de peso o cardioprotección versus restricción calórica diaria, aunque el AI puede ser más efectivo para no perder la masa magra.

Por otro lado, recientemente una revisión sistemática y un metaanálisis que incluyó estudios de cohortes y transversales examinó el cambio de peso al consumir $\mathrm{u}$ omitir el desayuno. Los autores encontraron que omitir el desayuno aumenta el riesgo de sobrepeso/ obesidad en $48 \%$ en estudios transversales y $44 \%$ en estudios de cohorte ${ }^{(20)}$.

En segundo lugar, omitir el desayuno aumentó el riesgo de obesidad abdominal en un $31 \%$ en estudios transversales. Se necesitan más estudios observacionales y de intervención para identificar los mecanismos subyacentes que vinculan la omisión del desayuno con el sobrepeso/obesidad.

\section{Enfermedades cardiovasculares}

El AI mejora múltiples indicadores de la salud cardiovascular tanto en animales como en humanos, incluyendo: presión sanguínea, la frecuencia cardíaca en reposo, colesterol, los niveles de lipoproteínas de alta y baja densidad (HDL-LDL) glucosa, insulina, resistencia a la insulina y triglicéridos.

El AI reduce los marcadores de inflamación sistémica y estrés oxidativo asociados a la aterosclerosis ${ }^{(21)}$.

Por otro lado, en un reciente estudio transversal con una muestra importante $(\mathrm{n}=4.052)$, reportó que los individuos que no desayunaron tuvieron mayor riesgo de aterosclerosis en comparación con aquellos que ingirieron altas calorías en el desayuno (> $20 \%$ calorías diarias) ${ }^{(22)}$. A pesar de esto, en los individuos que no desayunaron en comparación con el grupo de ingesta calórica elevada, se evidenciaron parámetros desfavorables: mayor porcentaje de obesidad central, peso corporal, índice de masa corporal, circunferencia de cintura, dislipidemia y glucemia. Los pacientes eran mayores, con un elevado porcentaje de mujeres y fumadoras, que habían ingerido una alimentación con un mayor aporte de calorías por día, proteínas animales, grasas totales, colesterol, alimentos procesados, bebidas alcohólicas y por otro lado, consumieron menos aporte de fibra dietética, vegetales y granos enteros. Se necesitan ensayos con un mayor tamaño de pacientes para evaluar los efectos del AI principalmente en pacientes con dislipidemia. Es apresurado mencionar que la privación del desayuno conduce a eventos cardiovasculares.

\section{Cáncer}

La evidencia muestra que el ayuno protege de la toxicidad al tiempo que mejora la eficacia de una variedad de quimioterapéuticos en el tratamiento de varios tipos de tumores ${ }^{(23)}$.

Estudios a largo plazo realizados en humanos con restricción calórica, mostraron una reducción del metabolismo, hormonas y factores asociados al riesgo de cáncer. Sin embargo, la restricción calórica a largo plazo, no es una intervención clínica factible. Los estudios preclínicos sugieren que el ayuno protege a los roedores de los efectos tóxicos de la quimioterapia, mientras que aumenta, simultáneamente, la eficacia de una variedad de agentes quimioterápicos en numerosas neoplasias malignas ${ }^{(24-26)}$.

Los bajos niveles de glucosa en suero durante el ayuno imponen un estrés adicional en las células tumorales, ya que las necesidades energéticas en estas circunstancias se satisfacen principalmente por medio de la glucólisis.

De manera similar, se cree que el AI perjudica el metabolismo energético en las células cancerosas inhibiendo su crecimiento y haciéndolas susceptibles a tratamientos clínicos ${ }^{(21)}$.

El papel de la autofagia en el cáncer es complejo, y su función puede variar de acuerdo con factores biológicos, incluido el tipo de tumor, y la etapa de progresión. La autofagia puede utilizarse como objetivo terapéutico en el tratamiento de diferentes tumores, y una nueva estrategia en el manejo de algunos tumores malignos ${ }^{(27)}$. El ayuno, entonces, puede proteger a las células normales de la toxicidad de los agentes anticancerígenos, reduciendo los efectos secundarios en los pacientes.

Varios estudios de casos en pacientes con glioblastoma sugieren que el AI puede suprimir el crecimiento tumoral y prolongar la supervivencia ${ }^{(3,28,29)}$.

El AI emerge como una estrategia prometedora para mejorar la eficacia y la tolerabilidad de la quimioterapia en el cáncer.

\section{Trastornos neurodegenerativos}

El excesivo aporte de energía a través de la ingesta de alimentos, particularmente en la mediana edad, aumenta los riesgos de accidente cerebrovascular, enfermedad de Alzheimer y enfermedad de Parkinson ${ }^{(1)}$.

El ayuno induce la alteración coordinada de muchos mecanismos metabólicos y transcripcionales, que puede influir en las neuronas. Colectivamente, estas alteraciones producen una alteración del estado meta- 
bólico que optimiza la bioenergética de las neuronas, la plasticidad y la resistencia al estrés, manteniendo o mejorando el rendimiento cognitivo ${ }^{(30)}$.

En ausencia de estudios específicos sobre este tema, muchas preguntas permanecen sobre la mejor forma de adaptar el tratamiento de la enfermedad de Parkinson durante el ayuno de Ramadán y el efecto de ese período en el control de la enfermedad a corto y largo plazo.

En el accidente cerebrovascular el ayuno reduce los niveles de factores proinflamatorios como la proteína C reactiva, IL-6 y homocisteína que pueden inhibir la formación de placas ateroscleróticas ${ }^{(31)}$.

Hay poca evidencia que apoya el ayuno para el control de las convulsiones en modelos de animales en la epilepsia. Algunos estudios en niños encontraron mejora en el control de las convulsiones más allá de una dieta cetogénica, y en ayunas. Sin embargo, el número limitado de estudios, tamaño de la muestra, resultó en evidencia deficiente y de mala calidad. En todos los estudios se encontraron efectos adversos como trastornos gastrointestinales relacionados a corto plazo, a complicaciones cardiovasculares a más largo plazo ${ }^{(32)}$.

\section{Esclerosis múltiple (EM)}

La EM es un trastorno inflamatorio, mediado por autoinmunidad que daña el sistema nervioso central y sus axones.

Los cuerpos cetónicos se producen durante el ayuno y son una fuente de energía alternativa y presumiblemente eficiente para el cerebro (BHB y acetoacetato).

Dos estudios recientes mostraron que los pacientes con EM que se adhieren al AI han reducido los síntomas en un período corto de 2 meses al disminuir la inflamación ${ }^{(33,34)}$.

\section{Asma}

La obesidad es un factor de riesgo para el asma y la pérdida de peso puede mejorar los síntomas ${ }^{(35,36)}$.

Un reciente metaanálisis encontró una relación dosisrespuesta entre la obesidad y el asma: la razón de probabilidad de incidencia de asma fue de 1,5 en sujetos con sobrepeso y 1,9 en obesos en comparación con el grupo delgado. La prevalencia de asma en mujeres delgadas versus obesas es de $7,9 \%$ y $14,6 \%$, respectivamente ${ }^{(37)}$.

\section{Artritis reumatoidea (AR)}

La reducción del dolor y la inflamación en la AR se ha relacionado con el ayuno, aunque también se conoce la reanudación de los síntomas luego de restablecer los hábitos de alimentación regulares. Los beneficios del ayuno en la $\mathrm{AR}$, si los hay, son transitorios y parecen no causar efectos a largo plazo en la actividad de la enfer$\operatorname{medad}^{(38,39)}$.

\section{CONCLUSIÓN Y CONSIDERACIONES PARA LA PRÁCTICA CLÍNICA}

A pesar de la evidencia científica de los beneficios del AI, para la aplicabilidad de este tipo de intervención dietética, se deberán tomar en cuenta las siguientes consideraciones:

1. Una dieta con 6 comidas está muy arraigada en nuestra cultura y un cambio en ese patrón de alimentación rara vez será contemplado por pacientes o profesionales.

2. Los estudios en roedores apoyan la hipótesis de que el AI y la restricción de alimentos durante la noche, permite mejorar los perfiles metabólicos y reducir los riesgos de obesidad, enfermedades crónicas, diabetes y cáncer.

3. Realizar este tipo de ayuno genera hambre, irritabilidad y una capacidad reducida para concentrarse durante los períodos de restricción de alimentos. Sin embargo, los efectos secundarios iniciales generalmente desaparecen dentro de un mes, pero los pacientes deben ser alertados de este hecho. En otros casos puede indicarse ayuno en períodos cortos e ir aumentando poco a poco el tiempo con el objetivo de que se adapte a esta práctica.

4. Los planes de alimentación de ayuno parecen promover la pérdida de peso y pueden mejorar el metabolismo. Sin embargo, se necesita más investigación clínica para evaluar este tipo de restricción energética, ayuno óptimo, tamaño muestral, número de días de ayuno por semana, grado de restricción energética necesaria en los días de ayuno y de ser así, si podría facilitar la mejoría metabólica a largo plazo.

5. Se debe garantizar que se satisfagan las necesidades nutricionales del paciente y proporcionar asesoramiento y educación alimentaria para evitar deficiencias nutricionales.

6. No está indicado este tipo de alimentación a personas que padecen o han padecido trastornos de la conducta alimentaria, tendencia al estrés y ansiedad.

\section{Financiación}

El presente trabajo no tuvo financiación. 


\section{Declaración de conflicto de intereses}

La autora declara no tener conflicto de intereses en relación con este artículo.

\section{Referencias bibliográficas}

1. Phillips MCL. Fasting as a Therapy in Neurological Disease. Nutrients. 2019; 11(10):2501. Doi: 10.3390/nu11102501.

2. de Cabo R, Mattson MP. Effects of Intermittent Fasting on Health, Aging, and Disease. N Engl J Med. 2019; 381(26):2541-51. doi: 10.1056/NEJMra1905136.

3. Puchalska P, Crawford PA. Multi-dimensional Roles of Ketone Bodies in Fuel Metabolism, Signaling, and Therapeutics. Cell Metab.2017;25(2):262-84. doi: 10.1016/j.cmet.2016.12.022.

4. Anton SD, Moehl K, Donahoo WT, Marosi K, Lee SA, Mainous AG, et al. Flipping the Metabolic Switch: Understanding and Applying the Health Benefits of Fasting. Obesity. 2018; 26(2):254-68. doi: 10.1002/oby.22065.

5. Patterson RE, Sears DD. Metabolic Effects of Intermittent Fasting. Annu Rev Nutr. 2017;37:371-93. doi: 10.1146/ annurev-nutr-071816-064634.

6. Bagherniya M, Butler AE, Barreto GE, Sahebkar A. The effect of fasting or calorie restriction on autophagy induction: a review of the literature. Ageing Res Rev. 2018;47:183-97. doi: 10.1016/j.arr.2018.08.004.

7. Potter GMD, Skene DJ, Arendt J, Cade JE, Grant PJ, Hardie LJ. Circadian Rhythm and Sleep Disruption: Causes, Metabolic Consequences, and Countermeasures. Endocr Rev. 2016; 37(6):584-608. doi: 10.1210/er.2016-1083.

8. Gamble KL, Berry R, Frank SJ, Young ME. Circadian Clock Control of Endocrine Factors. Nat Rev Endocrinol. 2014;10(8):466-75. Doi: 10.1038/nrendo.2014.78.

9. Gibbs M, Harrington D, Starkey S, Williams P, Hampton S. Diurnal postprandial responses to low and high glycaemic index mixed meals. Clin Nutr. 2014;33(5):889-94. doi: 10.1016/j.clnu.2013.09.018.

10. Potter GD, Cade JE, Grant PJ, Hardie LJ. Nutrition and the Circadian System. Br J Nutr. 2016;116(3):434-42. Doi: $10.1017 /$ S0007114516002117.

11. Varady KA, Bhutani S, Klempel MC, Kroeger CM, Trepanowski JF, Haus JM, et al. Alternate Day Fasting for Weight Loss in Normal Weight and Overweight Subjects: A Randomized Controlled Trial. Nutr J. 2013; 12(1):146. doi: 10.1186/1475-2891-12-146.

12. Herieka M, Erridge C. High-fat Meal Induced Postprandial Inflammation. Mol Nutr Food Res. 2014; 58(1):136-46. doi: 10.1002/mnfr.201300104.

13. Laugerette F, Alligier M, Bastard JP, Drai J, Chanséaume E, Lambert-Porcheron $S$, et al. Overfeeding Increases Postprandial Endotoxemia in Men: Inflammatory Outcome May Depend on LPS Transporters LBP and sCD14.
Mol Nutr Food Res. 2014;58(7):1513-8. doi: 10.1002/ mnfr.201400044.

14. Furmli S, Elmasry R, Ramos M, Fung J. Therapeutic Use of Intermittent Fasting for People With Type 2 Diabetes as an Alternative to Insulin. BMJ Case Rep. 2018: bcr2017221854. doi: 10.1136/bcr-2017-221854.

15. Sutton EF, Beyl R, Early KS, Cefalu WT, Ravussin E, Peterson CM. Early Time-Restricted Feeding Improves Insulin Sensitivity, Blood Pressure, and Oxidative Stress Even Without Weight Loss in Men With Prediabetes. Cell Metab. 2018; 27(6):1212-21.e3. doi: 10.1016/j.cmet.2018.04.010.

16. Harvie M, Howell A. Potential Benefits and Harms of Intermittent Energy Restriction and Intermittent Fasting Amongst Obese, Overweight and Normal Weight Subjects- A Narrative Review of Human and Animal Evidence. Behav Sci. 2017; 7(1); 4. doi: 10.3390 / bs7010004.

17. Harris L, Hamilton S, Azevedo LB, Olajide J, De Brún C, Waller $\mathrm{G}$, et al. Intermittent fasting interventions for treatment of overweight and obesity in adults: A systematic Review and Meta-Analysis. JBI Database System Rev and Implement Rep. 2018;16(2):507-47. doi: 10.11124/JBISRIR-2016-003248.

18. Varady KA. Intermittent Versus Daily Calorie Restriction: Which Diet Regimen Is More Effective for Weight Loss? Obes Rev. 2011;12(7):e593-601. doi: 10.1111/j.1467789X.2011.00873.x.

19. Trepanowski JF, Kroeger CM, Barnosky A, Klempel MC, Bhutani S, Hoddy KK, et al. Effect of Alternate-Day Fasting on Weight Loss, Weight Maintenance, and Cardioprotection Among Metabolically Healthy Obese Adults: A Randomized Clinical Trial. JAMA Intern Med. 2017; 177(7):930-8. doi: 10.1001/jamainternmed.2017.0936.

20. Ma X, Chen Q, Pu Y, Guo M, Jiang Z, Huang W, et al. Skipping Breakfast Is Associated With Overweight and Obesity: A Systematic Review and Meta-Analysis. Obes Res Clin Pract. 2020;14(1):1-8. Doi: https://doi.org/10.1016/j. orcp.2019.12.002.

21. Santos HO, Macedo RCO. Impact of Intermittent Fasting on the Lipid Profile: Assessment Associated With Diet and Weight Loss. Clin Nutr ESPEN. 2018; 24:14-21. doi: 10.1016/j.clnesp.2018.01.002.

22. Uzhova I, Fuster V, Fernández-Ortiz A, Ordovás JM, Sanz J, Fernández-Friera L, et al. The Importance of Breakfast in Atherosclerosis Disease: Insights From the PESA Study. J Am Coll Cardiol. 2017;70(15):1833-42. doi: 10.1016/j. jacc.2017.08.027.

23. de Groot S, Pijl H, van der Hoeven JJM, Kroep JR. Effects of Short-Term Fasting on Cancer Treatment. J Exp Clin Cancer Res. 2019;38(1):209. doi: 10.1186/s13046-019-1189-9.

24. Ravussin E, Redman LM, Rochon J, Das SK, Fontana L, Kraus WE, et al. A 2-Year Randomized Controlled Trial of Human Caloric Restriction: Feasibility and Effects on Predictors of Health Span and Longevity. J Gerontol A Biol Sci Med Sci. 2015; 70(9):1097-104. doi: 10.1093/gerona/glv057. 
25. Brandhorst $S$, Longo VD. Fasting and Caloric Restriction in Cancer Prevention and Treatment. Recent Results Cancer Res. 2016;207:241-66. doi: 10.1007/978-3-319-42118-6_12.

26. Antunes F, Erustes AG, Costa AJ, Nascimento AC, Bincoletto $\mathrm{C}$, Ureshino RP, et al. Autophagy and intermittent fasting: the connection for cancer therapy? Clinics. 2018;73:e814s. doi: $10.6061 /$ clinics/2018/e814s.

27. Kopeina GS, Senichkin VV, Zhivotovsky B. Caloric Restriction - A Promising Anti-Cancer Approach: From Molecular Mechanisms to Clinical Trials. Biochim Biophys Acta Rev Cancer. 2017;1867(1):29-41. doi: 10.1016/j. bbcan.2016.11.002.

28. Nencioni A, Caffa I, Cortellino S, Longo VD. Fasting and Cancer: Molecular Mechanisms and Clinical Application. Nat Rev Cancer. 2018; 18(11):707-19. doi: 10.1038/s41568-0180061-0.

29. Elsakka AMA, Bary MA, Abdelzaher E, Elnaggar M, Kalamian M, Mukherjee P, et al. Management of Glioblastoma Multiforme in a Patient Treated With Ketogenic Metabolic Therapy and Modified Standard of Care: A 24-Month Follow-Up. Front Nutr. 2018;5:20. doi: 10.3389 / fnut.2018.00020.

30. Mattson MP, Moehl K, Ghena N, Schmaedick M, Cheng A. Intermittent Metabolic Switching, Neuroplasticity and Brain Health. Nat Rev Neurosci. 2018; 19(2):63-80. doi: 10.1038/ nrn.2017.156.

31. Fann DYW, Ng GYQ, Poh L, Arumugam TV. Positive effects of intermittent fasting in ischemic stroke. Exp Gerontol. 2017;89:93-102. doi: 0.1016/j.exger.2017.01.014.

32. Martin K, Jackson CF, Levy RG, Cooper PN. Ketogenic diet and other dietary treatments for epilepsy. Cochrane Database
Syst Rev. 2016;2: CD001903. doi: 10.1002 / 14651858. CD001903.pub3.

33. Choi IY, Piccio L, Childress P, Bollman B, Ghosh A, Brandhorst S, et al. A Diet Mimicking Fasting Promotes Regeneration and Reduces Autoimmunity and Multiple Sclerosis Symptoms. Cell Rep. 2016;15(10):2136-46. doi: 10.1016/j.celrep.2016.05.009.

34. Fitzgerald KC, Vizthum D, Henry-Barron B, Schweitzer A, Cassard SD, Kossoff E, et al. Effect of Intermittent vs. Daily Calorie Restriction on Changes in Weight and PatientReported Outcomes in People With Multiple Sclerosis. Mult Scler Relat Disord. 2018;23:33-9. doi: 10.1016/j. msard.2018.05.002.

35. Carpaij OA, van den Berge M. The Asthma-Obesity Relationship: Underlying Mechanisms and Treatment Implications. Curr Opin Pulm Med. 2018;24(1):42-9. doi: 10.1097/MCP.0000000000000446.

36. Mohan A, Grace J, Wang BR, Lugogo N. The Effects of Obesity in Asthma. Curr Allergy Asthma Rep. 2019; 19(10):49. doi: 10.1007 / s11882-019-0877-z.

37. Peters U, Dixon AE, Forno E. Obesity and Asthma. J Allergy Clin Immunol. 2018;141(4):1169-78. Doi: 10.1007/s11882019-0877-z.

38. Badsha H. Role of Diet in Influencing Rheumatoid Arthritis Disease Activity. Open Rheumatol J. 2018;12:19-28. doi: 10.2174/1874312901812010019.

39. Longo VD, Mattson MP. Fasting: Molecular mechanisms and clinical applications. Cell Metab. 2014;19(2):181-92. doi: 10.1016/j.cmet.2013.12.008. 